\title{
Prognosis of acute respiratory distress syndrome in neutropenic cancer patients
}

\author{
Djamel Mokart*, Thomas van Craenenbroeck*, Jérôme Lambert*, Julien Textoris*, \\ Jean-Paul Brun*, Antoine Sannini*, Laurent Chow-Chine*, Smail Hamouda*, \\ Louis Fouché*, Florence Ettori*, Marion Faucher* and Jean-Louis Blache*
}

ABSTRACT: To date, no study has been specifically designed to identify determinants of death in neutropenic cancer patients presenting with acute respiratory distress syndrome (ARDS). The aim of this study was to identify early predictive factors of 28-day mortality in these patients. Factors associated with 28-day mortality during intensive care unit (ICU) stay were also described.

70 consecutive cancer patients with ARDS and neutropenia were prospectively analysed over a 6-yr period.

Mortality at 28 days was $63 \%$. Factors independently associated with good prognosis were: lobar ARDS (OR $0.10,95 \% \mathrm{Cl} 0.02-0.48$ ), use of initial antibiotic treatment active on difficult to treat bacteria (ticarcillin-resistant Pseudomonas aeruginosa, Stenotrophomonas maltophilia or extended-spectrum $\beta$-lactamase-producing strains) (OR $0.08,95 \% \mathrm{Cl} 0.02-0.33$ ) and first-line chemotherapy (OR $0.08,95 \% \mathrm{Cl} 0.02-0.37$ ). During the ICU stay, mortality was associated with the markers of organ dysfunctions, the absence of neutropenia recovery and the use of vasopressors. During the first 3 weeks, the conditional probability of discharge alive from ICU did not decrease.

At ICU admission, first-line chemotherapy, lobar ARDS and antibiotic treatment active on difficult-to-treat bacteria were associated with survival. During ICU stay, mortality was associated with organ dysfunctions and use of vasopressors. Most survivors have an ICU stay of $>3$ weeks.

KEYWORDS: Acute respiratory distress syndrome, cancer, neutropenia, prognosis

$\mathbf{R}$ ecent advances in the treatment of solid and haematological malignancies have led to a significant decrease over time in overall mortality in cancer patients [1]. The price to pay for increasing treatment intensity and duration, with the goal to improving survival, is a noteable rise in treatment-related toxicities and susceptibility to infection [2]; patients with neutropenia are vunerable to both conditions. In patients with febrile neutropenia, lung involvement is the predominant complication, with $20 \%$ of patients presenting with lung infiltrates [3] and $15 \%$ of patients presenting with respiratory events [4]. Under these circumstances, neutropenic patients are at high risk of developing acute respiratory distress syndrome (ARDS). Studies involving ARDS in neutropenic patients are scarce [5], but knowledge regarding cancer patients admitted to the intensive care unit (ICU) for acute respiratory failure (ARF) is now abundant and instructive. Approximately $15 \%$ of cancer patients develop ARF requiring an admission to ICU [6]. Survival of cancer patients presenting with ARF has improved over time to $60 \%$ [6]. These patients carry a hospital mortality rate of about $75 \%$ when they are ventilated [7]. No published study has been designed specifically to identify determinants of death in critically ill neutropenic cancer patients with ARDS. We sought to identify early predictive factors of 28-day mortality in 70 cancer patients presenting ARDS during chemotherapy-induced neutropenia. Factors associated with 28-day mortality during ICU stay were also described.

\section{PATIENTS AND METHODS}

The Paoli-Calmettes Institute, Marseille, France, is a 207-bed cancer referral centre, where $\sim 10,000$ patients (70\% medical and 30\% surgical) with a diagnosis of malignancy are hospitalised each year. All consecutive patients with haematological malignancy or solid tumours admitted to our medical-surgical ICU between January 1, 2002, and December 31, 2008, were evaluated prospectively. Only patients admitted for ARDS with a neutropenia were included in our study. The study was approved by our institutional review board (Paoli-Calmette Institute, Marseille, France), which waived the need for informed consent.

\section{AFFILIATIONS}

*Intensive Care Unit, Paoli-Calmette Institute, Marseille, and

\#Département Biostatistique et Informatique Médicale, Hôpital Saint Louis, Université Paris VII, Paris, France.

\section{CORRESPONDENCE}

D. Mokart

Intensive Care Unit Paoli-Calmette Institute 232 bd Sainte Marguerite 13273 Marseille Cedex 9 France E-mail: mokartd@ipc.unicancer.fr

Received:

Aug 312011 Accepted after revision: Nov 142011 First published online: Dec 012011 


\section{Definitions}

We used the definition of ARDS recommended by the AmericanEuropean Consensus Conference [8] and sepsis was defined according to the criteria of the American College of Chest Physicians/Society of Critical Care Medicine Consensus Conference [9]. "Diffuse", "lobar" and "patchy" ARDS were diagnosed using previously reported criteria [10], as were pulmonary and extrapulmonary ARDS [11]. Severe neutropenia was defined as absolute neutrophils count $<500$ cells per $\mu \mathrm{L}$.

\section{Criteria for ICU admission}

In our institution, ICU admission policy is broad and includes most patients with a "therapeutic project", regardless of their cancer status. Decisions to admit patients to the ICU for ARF were made by both referring oncologists or haematologists and a senior intensive care specialist. ARF was defined as oxygen saturation $<90 \%$ or arterial oxygen tension $\left(\mathrm{Pa}_{\mathrm{a}} \mathrm{O}_{2}\right)<60 \mathrm{mmHg}$ on room air combined with severe dyspnoea at rest with inability to speak in sentences or respiratory frequency $>30$ breaths per min or clinical signs of respiratory distress (recruitment of accessory respiratory muscles or respiratory muscle exhaustion). When possible, noninvasive mechanical ventilation (NIMV) was delivered to the patient through a full-face mask, and, in all cases, NIMV was performed only in the ICU. Patients considered to benefit from immediate ICU admission were managed systematically and daily by both the intensive care specialist and the haematologist/oncologist until ICU admission. During the ICU stay the referring haematologists/oncologists were systematically involved in all medical decisions regarding cancer evaluation, cancer treatment and treatment limitations.

\section{Patient's management}

Before ICU admission all patients underwent chest radiography and high-resolution computed tomography (HRCT) of the lung as recommended [12]. Just after ICU admission and as recommended by guidelines $[13,14]$, initial antibiotic treatment was reconsidered and all patients underwent broad-spectrum antibiotic treatment including at least an antipseudomonal $\beta$ lactam immediately after clinical evaluation and the realisation of microbiological samples. Beside antibiotics, most of patients underwent standardised supportive care according to the surviving sepsis campaign [15] and ARDS guidelines [16].

\section{Diagnostic strategy in neutropenic patients with ARDS}

Diagnoses were based on clinical, radiographic and microbiological findings, and were validated by the ICU team. Aetiological diagnoses of ARF were established by the physicians according to standardised definitions [7]. All patients were initially treated as a clinically documented infection (presence of a clinical or radiological infectious focus, or both, without causative pathogen identification). Microbiologically documented infection was defined as either a presence of a clinical or radiological infectious focus, or both, and pathogen identification. Microbiologically documented infection was considered only when the infection was the main cause of ARDS. Pneumocystis pneumonia (PCP) was diagnosed by the detection of Pneumocystis jiroveci using conventional staining methods (direct and indirect fluorescence antibody and toluidine-blue-O, Grocott-Gomori silver stain or Giemsa stains) in respiratory samples such as induced sputum or bronchoalveolar lavage fluid.
In our institution, difficult-to-treat (DTT) bacteria were represented by a group of pathogens including ticarcillinresistant Pseudomonas aeruginosa, Stenotrophomonas maltophilia or, rarely, extended-spectrum $\beta$-lactamase (ESBL)-producing strains. At ICU admission, initial antibiotic treatment active on DTT bacteria was defined as an antibiotic treatment usually active, in our institution, on the pre-defined DTT bacteria.

\section{Data collection}

Patient's characteristics were collected prospectively and are reported in tables 1 and 2. Early predictive factors for 28-day mortality were collected at ICU admission (day 1), and were defined as factors available at ICU admission such as initial characteristics of the patient, the underlying malignancy and factors regarding the ARDS, which included age, sex, absence of comorbidity, underlying malignancies, delay since malignancy at ICU admission (days), status of malignancy, bone marrow transplantation recipients, delay since neutropenia at ICU admission (days), organ dysfunctions using the logistic organ dysfunction (LOD) score and the Simplified Acute Physiology Score (SAPS) II score, arterial lactates concentration, causes of ARDS, lung morphology on computed tomography scan, occurrence of pulmonary or extrapulmonary ARDS, existence of microbiological documentation at ICU admission and antimicrobial treatment. Other factors associated with 28-day mortality were also collected during the ICU stay. Organ dysfunctions were reported according to the LOD score. To assess the impact of the LOD score change between day 1 and day 7 , we constructed a continuous variable by computing the difference (LOD day 7-LOD day $1)=\Delta \mathrm{LOD}$. Patients presenting a $\Delta \mathrm{LOD}>1$ were considered as clinically worsened, those presenting a score of $-1 \leqslant \Delta \mathrm{LOD}$ $\leqslant 1$ were considered clinically stable, and those with a score of $\Delta \mathrm{LOD}<-1$ were considered clinically improved. The SAPS II score was collected at ICU admission (day 1), the LOD score and $\mathrm{Pa}, \mathrm{O}_{2} /$ inspiratory oxygen fraction ratio were collected at day 1 , day 3 , day 7 and 2 days after neutropenia recovery. Other therapeutic interventions, such as vasopressor use, need and duration of respiratory support NIMV or conventional mechanical ventilation, renal replacement therapy (RRT), duration of renal replacement and length of ICU stay were collected; ICU, 28-day, hospital and 1-yr mortalities were also collected.

\section{Statistical analysis}

All data are presented as frequencies (percentage) for qualitative variables and medians (interquartile ranges) for quantitative variables. Characteristics of patients at admission and during ICU hospitalisation were compared across the groups of day-28 survivors and decedents using Fisher's exact test and Wilcoxon's rank-sum test. A multivariable logistic regression model was performed to identify independent predictors of day-28 mortality among baseline characteristics. Due to the relatively small sample size, bootstrapping was used to ensure the robustness of the model selection procedure. More precisely, missing values were imputed via multiple imputations by chained equation [17] to obtain 30 imputed datasets. Then, 200 bootstrap samples were drawn from each of the 30 imputed datasets, by random sampling with replacement. This led to 6,000 bootstrapped datasets in which a forward-stepwise Akaike information 
TABLE 1 Patients characteristics at intensive care unit (ICU) admission

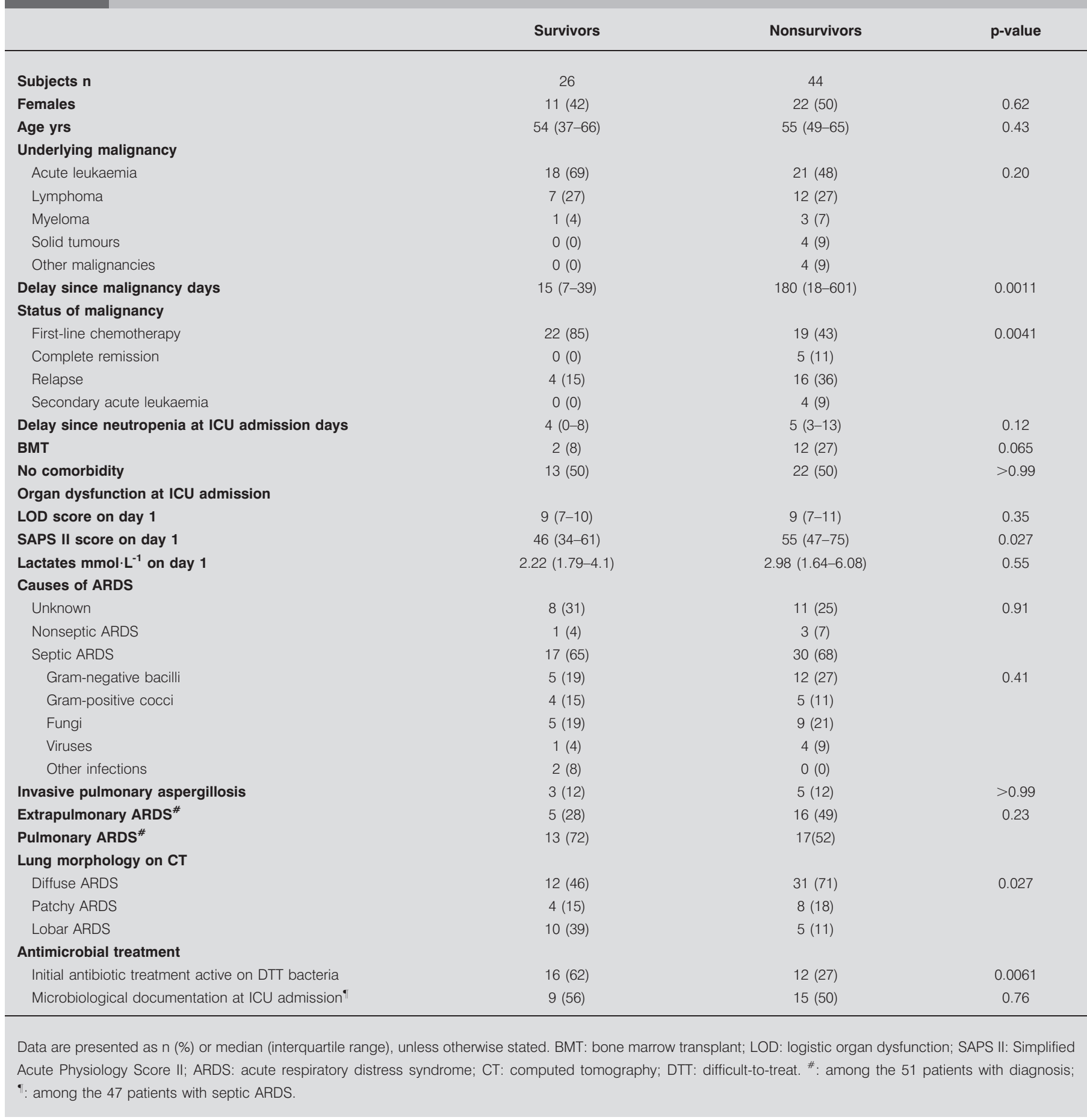

criterion (AIC)-based selection was repeated. This selection included all the baseline characteristics easily available at ICU admission that were significant at a $\mathrm{p}<0.10$ level in the original univariate analysis. Predictors that were selected in $>80 \%$ of the selection procedures were kept in the final model. Log-linearity was checked and continuous variables were dichotomised if necessary by using a clinically relevant cut-off. Calibration was tested by the le Cessie-van Houwelingen goodness-of-fit test and discrimination was assessed by the C-index, which is equivalent to receiver-operating characteristic area under the curve. To study the influence of the length of stay in ICU on the prognosis, the transition probability of discharge alive from ICU was estimated via multistate modelling, i.e. for each time $t$, the probability that a patient still in ICU at $t$ will eventually be discharged alive. All tests were two-sided, and statistical analysis was performed with R version 2.9.2 (R Development Core Team 2011; R Foundation for Statistical Computing, Vienna, Austria). 


\section{TABLE 2 Patient characteristics during intensive care unit stay}

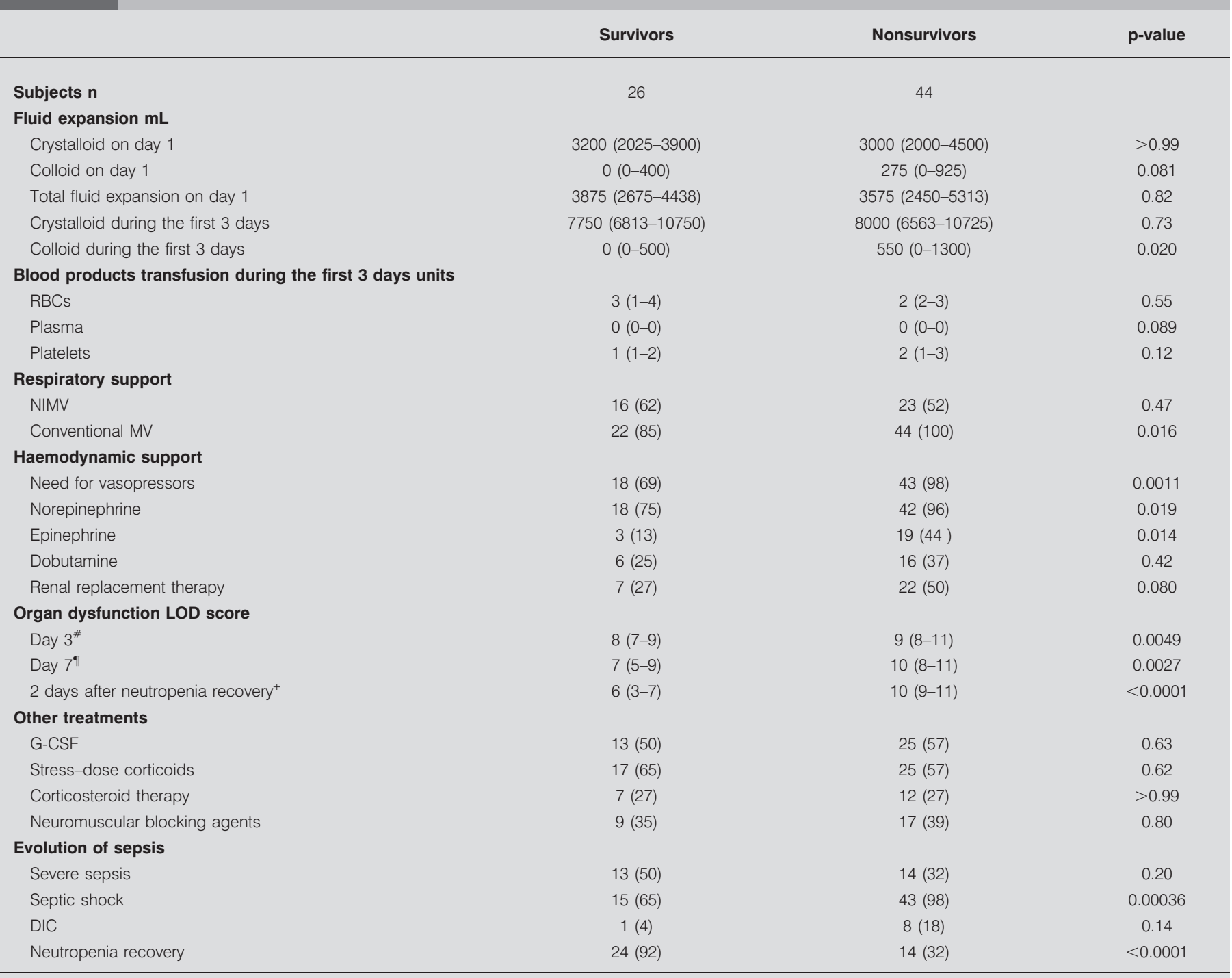

Data are presented as median (interquartile range) or $\mathrm{n}(\%)$, unless otherwise stated. RBC: red blood cell; NIMV: noninvasive mechanical ventilation; MV: mechanical ventilation; LOD: logistic organ dysfunction; G-CSF: granulocyte colony-stimulating factor; DIC: disseminated intravascular coagulation. ${ }^{\#}$ : among the 61 patients alive at day $3 ;{ }^{\bullet}$ : among the 52 patients alive at day $7 ;^{+}$: among the 38 patients with neutropenia recovery.

\section{RESULTS}

Among the 52,223 medical patients admitted to the oncology and haematology wards at our institution during the study period, 70 were admitted to our ICU for neutropenic ARDS (fig. 1). Characteristics of patients are reported in tables 1 and 2. Of the $14(20 \%)$ bone marrow transplant (BMT) recipients, nine had received autologous BMT and five allogeneic BMT. During the ICU course, granulocyte colony-stimulating factor was used in $38(54 \%)$ patients, for a median of 5 (interquartile range 2-11) days.

\section{At ICU admission}

All patients received antibiotics on admission to ICU. $\beta$-lactams were used in all patients; vancomycin in $56(80 \%)$, antifungal agents in 41 (71\%), quinolones in $43(61 \%)$, trimethoprimsulfamethoxazole (TMP-SMX) in 25 (38\%), aminoglycosides in
$17(24 \%)$ and antiviral agents in 16 patients (23\%). $65(93 \%)$ patients received three or more anti-infectious agents. The 28 $(40 \%)$ patients already treated with initial antibiotic treatment active on DTT bacteria at ICU admission showed a lower, although nonsignificant, microbiologically documented infection rate $(15(54 \%)$ out of 28 versus 31 (74\%) out of 42 , respectively; $\mathrm{p}=0.12)$. In this group of patients and in all cases, treatment was associated with at least three antibiotics. Among these antibiotics, imipenem/cilastatin was used in $24(86 \%)$ patients, TMP-SMX in 25 (89\%), ceftazidime in five (18\%) and colistin in three (11\%). Interestingly, imipenem/cilastatin and TMP-SMX were associated with 22 (79\%) patients. DTT bacteria were documented in 13 patients (19\%). Invasive pulmonary aspergillosis was diagnosed in eight patients and PCP in two patients. Mortality rates of patients with and without invasive pulmonary aspergillosis were not different (five out of eight 


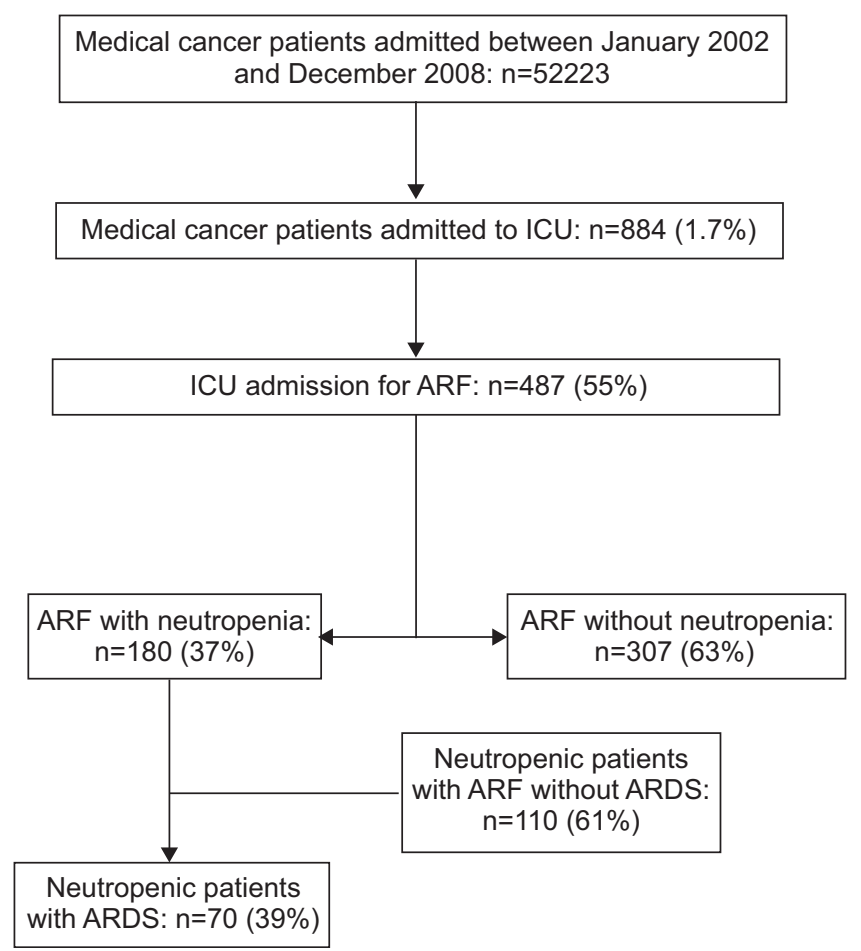

FIGURE 1. Flow diagram of study. ARDS: acute respiratory distress syndrome; ARF: acute respiratory failure; ICU: intensive care unit.

deaths at day 28 versus 39 out of $62 ; \mathrm{p}>0.99$ ) (table 1 ). At ICU admission, patients who exhibited subsequent neutropenia recovery showed similar SAPS II and LOD scores compared with patients without neutropenia recovery (median 51 (range 42-65) versus 54 (43-82); $\mathrm{p}=0.45$ and 9 (7-10) versus 10 (7-12); $p=0.20$, respectively). 41 patients received first-line chemotherapy, as follows. 30 out of the 41 patients with acute leukaemia, eight out of the 19 patients with lymphoma, one out of the four patients with myeloma, none of the four patients with solid tumour and two out of the four patients with other

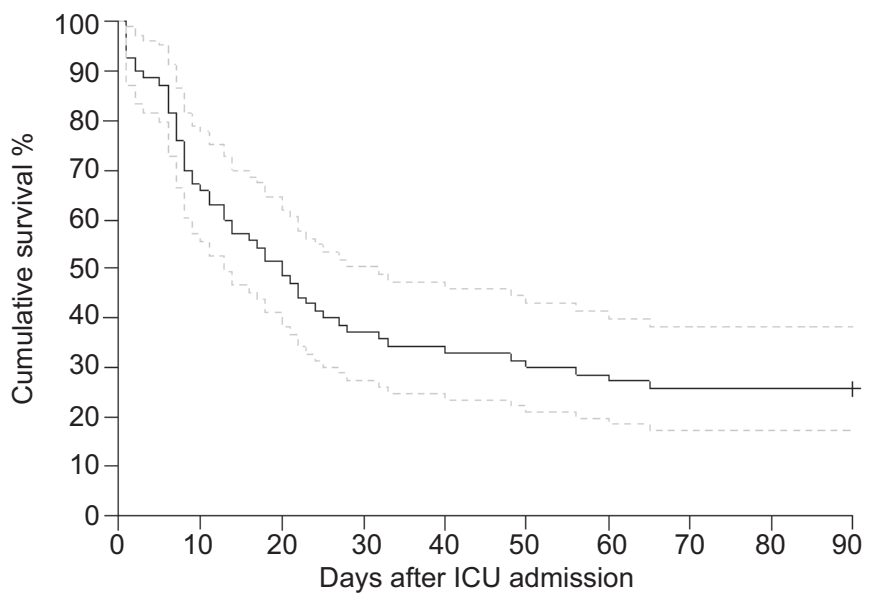

FIGURE 2. Survival in neutropenic patients with aquired respiratory distress sydrome. Overall survival is truncated at 90 days. -----: $95 \%$ confidence interval. ICU: intensive care unit. malignancies. Thus, no patient with solid tumour received firstline chemotherapy.

\section{During ICU stay}

Table 2 compares patient characteristics and ICU therapy between day-28 survivors and decedents. Beside ARDS, 53 $(76 \%)$ patients developed a renal dysfunction, $25(36 \%)$ developed a hepatic dysfunction and six $(8.6 \%)$ presented a neurological dysfunction. Vasopressors were needed in 61 $(87 \%)$ patients and RRT in $29(41 \%)$, and $66(94 \%)$ patients received mechanical ventilation, the duration of which was median 10 (range 6-22) days. The ICU length of stay was 16 (7$25)$ days. Mortality at day 28 was $44(63 \%)$ out of 70 patients; hospital mortality and ICU mortality were identical at about $73 \%$ (51 out of 70 patients). Within the first 10 days of ICU admission, mortality rate was $\sim 33 \%$ (fig. 2 ) and only $11 \%$ of ICU survivors (two patients) had left the ICU (fig. 3a). Among the 52 patients still alive in ICU at day 7, the clinical status of 13 worsened during the first week of ICU stay, 25 remained stable and 14 improved their clinical status. Day 28 mortality for these three categories was nine out of $13(69 \%), 13$ out of 25 $(52 \%)$ and five out of $14(36 \%)$ (Chi-squared test for trend, $\mathrm{p}=0.08$ ). Among the 19 patients who were discharged alive
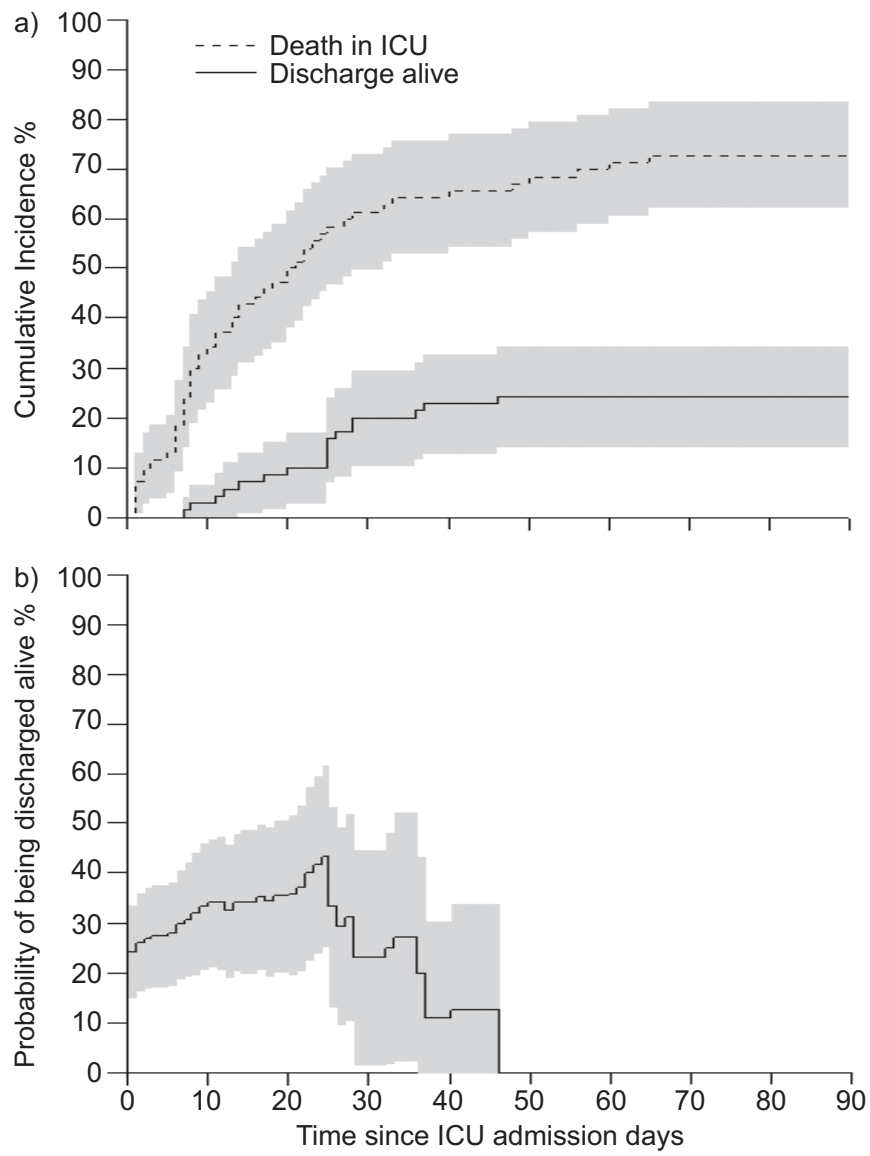

Subjects at risk $\mathrm{n}$

$\begin{array}{llllllllll}70 & 45 & 28 & 13 & 9 & 6 & 4 & 2 & 2 & 2\end{array}$

FIGURE 3. a) The cumulative incidence of death in intensive care unit (ICU) and discharge alive. b) The daily transition probabilities between ICU and discharge alive. Shaded area represents 95\% confidence interval. 

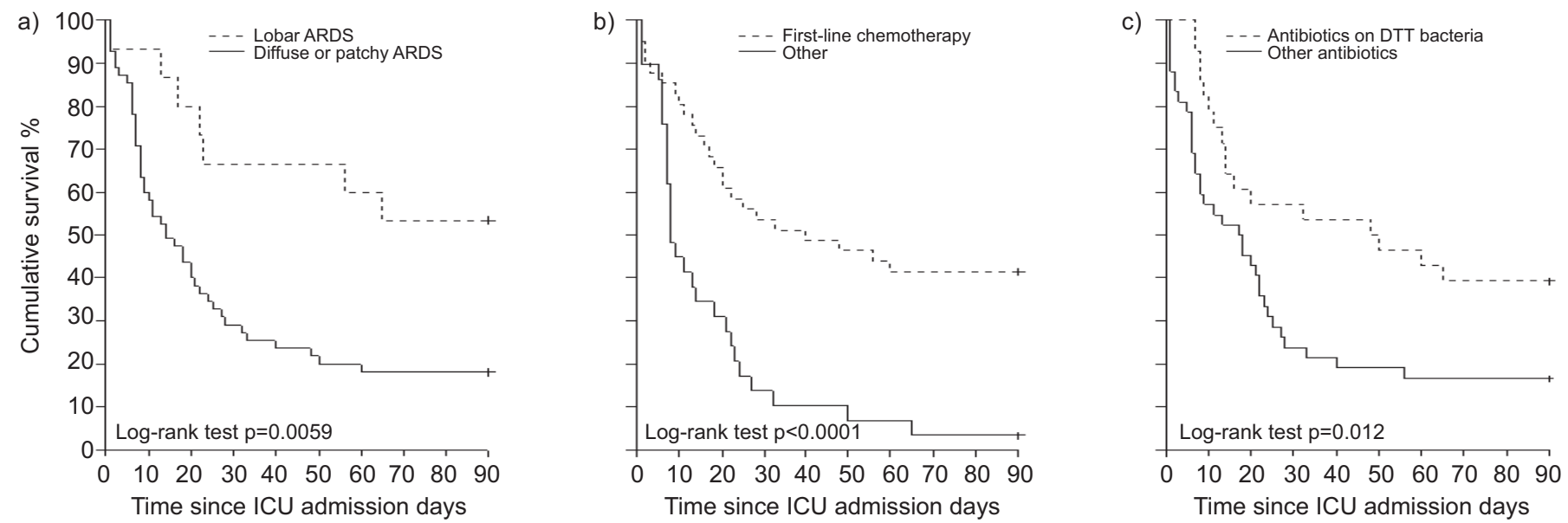

FIGURE 4. Cumulative incidence of death in intensive care unit (ICU) and discharge alive from ICU according to a) lung morphology on computed tomography, b) status of malignancy and c) initial antimicrobial treatment. ARDS: acute respiratory distress syndrome; DTT: difficult-to-treat.

from ICU, the median length of stay was 25 days (range 7120 days) (fig. 3a). 17 (89\%) of these 19 patients recovered from neutropenia before leaving the ICU within a median of 11 days. Among the 62 survivors present in ICU at day 3, 26 (42\%) were still alive at day 28; among the 52 survivors present in ICU at day 7, 25 (48\%) were still alive at day 28; and among the 26 patients still alive at day 28 , eight finally died in ICU after $>1$ month of ICU hospitalisation. 19 patients were discharged alive from ICU and, among them, 16 received chemotherapy; 14 of these 16 patients achieved a complete remission and nine out of these 14 patients were still alive at $1 \mathrm{yr}$.

The transition probability between ICU and discharge alive was represented over time. For each day $n$, this probability represents the proportion of all patients still alive in ICU after $n$ days who will finally be discharged alive. During the first 3 weeks, transition probabilities were flat or even increasing (fig. 3b), indicating that patients with longer length of stay were not at a higher risk of death in ICU.

The results of the univariate analysis at ICU admission are shown in table 1 . The SAPS II score, time from diagnosis of cancer, first-line chemotherapy, use of initial antibiotic treatment active on DTT bacteria and lobar ARDS appeared to be related to day-28 mortality. Three factors were independently associated with a good prognosis in multivariate analysis (fig. 4 and table 3): 1) lobar ARDS (OR 0.10, 95\% CI 0.02-0.48); 2) use of initial antibiotic treatment active on DTT bacteria (OR $0.08,95 \%$ CI 0.02-0.33); and 3) first-line chemotherapy (OR $0.08,95 \%$ CI $0.02-0.37)$.

\section{DISCUSSION}

We report herein on 70 neutropenic patients admitted to ICU for ARDS over 6 yrs. From ICU admission, first-line chemotherapy, lobar ARDS and use of antibiotic treatment active on DTT bacteria were independently associated with 28-day survival. During the ICU stay, mortality was closely associated with the markers of organ dysfunctions, the absence of neutropenia recovery and use of vasopressors. Most survivors had an ICU stay of $>3$ weeks.
In critically ill neutropenic patients, we confirmed that neither the characteristics of the underlying malignancy nor age were associated with survival [18]. Interestingly, in this situation, first-line chemotherapy was protective. This result suggests a better prognosis for the patients with a recent cancer diagnosis and being newly treated [19].

Similarly to the general population [20], we found a strong association between mortality and lung morphology just before ICU admission. We showed that the "lobar ARDS" group represented $\sim 21 \%$ of our patients and was associated with a good prognosis. Strikingly, most of our patients presented patchy or diffuse lung attenuations (79\%), which are known to be associated with high mortality [20]. It has been shown that chest radiography provides a limited yield for the early detection of asymptomatic pneumonia in neutropenic patients [21] and early detection of lung infiltrates in febrile neutropenic patients seems to be more efficient using HRCT than chest radiography [12]. At the stage of obvious lung infiltrates on chest radiography, the infectious/inflammatory process could already be well advanced. Thus, our results suggest that an

\begin{tabular}{|c|c|c|}
\hline & OR $(95 \% \mathrm{Cl})$ & p-value \\
\hline Lobar ARDS & $0.10(0.02-0.48)$ & 0.0038 \\
\hline $\begin{array}{l}\text { Initial antibiotic treatment active } \\
\text { on DTT bacteria }\end{array}$ & $0.08(0.02-0.33)$ & 0.0007 \\
\hline First-line chemotherapy & $0.08(0.02-0.37)$ & 0.0014 \\
\hline
\end{tabular}

Variables included in this multivariate analysis with bootstrapped stepwise selection were simultaneously statistically significant at a p-value $<0.10$ in the univariate analysis and clinically relevant, i.e. easily available upon admission to the intensive care unit (ICU) (delay since malignancy, first-line chemotherapy as status of malignancy, bone marrow transplantation, lobar acute respiratory distress syndrome (ARDS) on computed tomography, Simplified Acute Physiology Score II score on day one, and use of antibiotic active on difficultto-treat (DTT) bacteria at ICU admission). 
early detection of focal lung infiltrates could have an impact on outcome and should be evaluated.

Interestingly, patients who were being treated with initial antibiotic treatment active on DTT bacteria at ICU admission showed a better prognosis. In this group of patients, no death occurred until the first week in ICU, whereas the mortality was $\sim 35 \%$ during this period for the others. These results suggest that an earlier implementation of an effective therapy may result in improved outcome [22]. Cancer patients with prolonged neutropenia, exposure to broad-spectrum antibiotics and those requiring mechanical ventilation have higher risk for S. maltophilia infection [23]. Similarly, the development of resistance to antimicrobial agents is well recognised in febrile neutropenic patients, in particular with antibiotic-resistant mutant strains that produce ESBLs [24]. Inadequate initial antimicrobial therapy, infection caused by multi-resistant bacteria and prolonged neutropenia are known to be independently associated with worse outcomes in haematology patients [24]. Accordingly, 19\% of our patients were infected by an aetiologically significant pathogen defined as DTT bacteria. In this situation, an initial empirical antibiotic treatment of active DTT bacteria could have had a positive effect on outcome. In fact, antibiotics active on $P$. aeruginosa are not always efficient on documented infections with S. maltophilia or ESBL-producing strains, and their combination with antibiotics such as TMP-SMX [23] or colistin may either extend their spectrum or act synergistically [25]. Our data suggest that, first, when considering that data on antibiotic resistance is dynamic and varies from ward to ward [26], broad-spectrum antibiotic treatment must be initiated promptly [22] and, secondly, the monitoring of patient colonisation status in order to permit a surveillance-assisted initiation of empirical antibiotics should be evaluated in such patients [27].

Among the 44 patients who did not survive at day 28, 42 (95\%) did not develop neutropenia recovery; this underlines the impact of persistent immunosuppression on outcome [28]. Accordingly, ICU admission of neutropenic cancer patients with ARF during neutropenia recovery is known to be associated with good prognosis [7]. After neutropenia recovery, the persistence of organ failure was significantly associated with ICU mortality. It is, therefore, crucial to know whether patients died too quickly to exhibit neutropenia recovery or if persistent neutropenia is a risk factor for death. SAPS II and LOD scores at ICU admission were similar in patients with and without subsequent neutropenia recovery, favouring the latter explanation.

For most patients with ARDS in the general population, their outcome is determined in 10 days, by which time about half the patients have either died or have been weaned off treatment [29]. In neutropenic patients, the course of ARDS seems to be quite different as only one-third of our patients had their outcome fixed at day 10 . It is noteworthy that $85 \%$ of ICU survivors (16 patients) were still hospitalised in ICU after the first 10 days of ARDS. Strikingly, the probability of being discharged alive from ICU reached a maximum at 24 days and then decreased. Nevertheless, this should not be overinterpreted due to the small sample size. After 4 weeks, 13 $(<20 \%)$ of the patients were still alive in the ICU and the uncertainty on the estimate of the transition is great, as illustrated by the wide $95 \%$ confidence interval. Thus, we can only state that there is no increase in the risk of death with the length of stay in the first 3 weeks of ICU stay.

Similarly to the overall cancer population, during ICU stay, the prognosis of the neutropenic cancer patients was associated with the persistence of organ failures and use of vasopressors. At the moment, ICU admission with full-code management followed by reappraisal on day 6 is recommended for cancer patients requiring mechanical ventilation [30]. As suggested by our results, an intensive management of neutropenic patients could be maintained for longer than 6 or 10 days. In fact, the conditional probability of discharge alive from ICU increased during the first 25 days. Most of our survivors had an ICU stay $>3$ weeks. Importantly, the interesting results in the survivor's length of stay do not rely on their comparison with deceased patients but rather in its absolute value. No patient was discharged alive before day 7 , and two-thirds of the survivors needed $>3$ weeks of ICU treatment before they could be discharged.

Interestingly, after the first week of ICU stay, $\sim 50 \%$ of patients who remained clinically stable, even in the absence of real clinical improvement, were still alive on day 28. Accordingly, $64 \%$ of patients who showed a clinical improvement during this period were also still alive on day 28 , whereas only $31 \%$ of patients who worsened their clinical status survived at day 28 . In these situations, mechanical ventilation and the use of vasopressors were clearly associated with day-28 mortality. These results suggest that all neutropenic patients with ARDS should benefit from initial full-code ICU management, including mechanical ventilation, use of vasopressors and renal replacement therapy. From our experience, life-sustaining therapies should be probably prolonged during at least 3 weeks in all patients presenting an improved or stable clinical status. A full ICU management, followed by reappraisal after 3 weeks should be evaluated in all neutropenic patients with ARDS.

\section{Conclusion}

At ICU admission, first-line chemotherapy, lobar ARDS and use of antibiotic treatment active on DTT bacteria are associated with survival. During ICU stay, neutropenia recovery seems to be an important appointment, whereas the persistence of organ failures and the use of vasopressors are associated with death. Most survivors have an ICU stay $>3$ weeks.

\section{STATEMENT OF INTEREST}

None declared.

\section{REFERENCES}

1 Brenner H, Gondos A, Arndt V. Recent major progress in longterm cancer patient survival disclosed by modeled period analysis. J Clin Oncol 2007; 25: 3274-3280.

2 Talpaz M, Shah NP, Kantarjian H, et al. Dasatinib in imatinibresistant Philadelphia chromosome-positive leukemias. $N$ Engl J Med 2006; 354: 2531-2541.

3 Rossini F, Verga M, Pioltelli P, et al. Incidence and outcome of pneumonia in patients with acute leukemia receiving first induction therapy with anthracycline-containing regimens. Haematologica 2000; 85: 1255-1260.

4 Puig N, De La Rubia J, Jarque I, et al. Characteristics of and risk factors for pneumonia in patients with hematological malignancies 
developing fever after autologous blood stem cell transplantation. Leuk Lymphoma 2007; 48: 2367-2374.

5 Ognibene FP, Martin SE, Parker MM, et al. Adult respiratory distress syndrome in patients with severe neutropenia. $N$ Engl $J$ Med 1986; 315: 547-551.

6 Azoulay E, Schlemmer B. Diagnostic strategy in cancer patients with acute respiratory failure. Intensive Care Med 2006; 32: 808-822.

7 Azoulay E, Mokart D, Rabbat A, et al. Diagnostic bronchoscopy in hematology and oncology patients with acute respiratory failure: prospective multicenter data. Crit Care Med 2008; 36: 100-107.

8 Bernard GR, Artigas A, Brigham KL, et al. The AmericanEuropean Consensus Conference on ARDS. Definitions, mechanisms, relevant outcomes, and clinical trial coordination. Am J Respir Crit Care Med 1994; 149: 818-824.

9 American College of Chest Physicians/Society of Critical Care Medicine Consensus Conference. Definitions for sepsis and organ failure and guidelines for the use of innovative therapies in sepsis. Crit Care Med 1992; 20: 864-874.

10 Puybasset L, Cluzel P, Gusman P, et al. Regional distribution of gas and tissue in acute respiratory distress syndrome. I. Consequences for lung morphology. CT Scan ARDS Study Group. Intensive Care Med 2000; 26: 857-869.

11 Agarwal R, Srinivas R, Nath A, et al. Is the mortality higher in the pulmonary vs the extrapulmonary ARDS? A meta analysis. Chest 2008; 133: 1463-1473.

12 Heussel CP, Kauczor HU, Heussel GE, et al. Pneumonia in febrile neutropenic patients and in bone marrow and blood stem-cell transplant recipients: use of high-resolution computed tomography. J Clin Oncol 1999; 17: 796-805.

13 Hughes WT, Armstrong D, Bodey GP, et al. 2002 guidelines for the use of antimicrobial agents in neutropenic patients with cancer. Clin Infect Dis 2002; 34: 730-751.

14 Mokart D, Sannini A, Brun JP, et al. Oncohematology patients with febrile neutropenia hospitalized in ICU: clinical practice guidelines. Réanimation 2008; 17: 213-224.

15 Dellinger RP, Carlet JM, Masur H, et al. Surviving Sepsis Campaign guidelines for management of severe sepsis and septic shock. Intensive Care Med 2004; 30: 536-555.

16 Brower RG, Lanken PN, MacIntyre N, et al. Higher versus lower positive end-expiratory pressures in patients with the acute respiratory distress syndrome. N Engl J Med 2004; 351: 327-336.

17 Rubin DB, Schenker N. Multiple imputation in health-care databases: an overview and some applications. Stat Med 1991; 10: 585-598.

18 Benoit DD, Vandewoude KH, Decruyenaere JM, et al. Outcome and early prognostic indicators in patients with a hematologic malignancy admitted to the intensive care unit for a lifethreatening complication. Crit Care Med 2003; 31: 104-112.

19 Vandijck DM, Benoit DD, Depuydt PO, et al. Impact of recent intravenous chemotherapy on outcome in severe sepsis and septic shock patients with hematological malignancies. Intensive Care Med 2008; 34: 847-855.

20 Rouby JJ, Puybasset L, Cluzel P, et al. Regional distribution of gas and tissue in acute respiratory distress syndrome. II. Physiological correlations and definition of an ARDS Severity Score. CT Scan ARDS Study Group. Intensive Care Med 2000; 26: 1046-1056.

21 Navigante AH, Cerchietti LC, Costantini $\mathrm{P}$, et al. Conventional chest radiography in the initial assessment of adult cancer patients with fever and neutropenia. Cancer Control 2002; 9: 346-351.

22 Lin MY, Weinstein RA, Hota B. Delay of active antimicrobial therapy and mortality among patients with bacteremia: impact of severe neutropenia. Antimicrob Agents Chemother 2008; 52: 3188-3194.

23 Safdar A, Rolston KV. Stenotrophomonas maltophilia: changing spectrum of a serious bacterial pathogen in patients with cancer. Clin Infect Dis 2007; 45: 1602-1609.

24 Trecarichi EM, Tumbarello M, Spanu T, et al. Incidence and clinical impact of extended-spectrum-beta-lactamase (ESBL) production and fluoroquinolone resistance in bloodstream infections caused by Escherichia coli in patients with hematological malignancies. J Infect 2009; 58: 299-307.

25 Kasiakou SK, Michalopoulos A, Soteriades ES, et al. Combination therapy with intravenous colistin for management of infections due to multidrug-resistant Gram-negative bacteria in patients without cystic fibrosis. Antimicrob Agents Chemother 2005; 49: 3136-3146.

26 Blot S, Depuydt P, Vogelaers D, et al. Colonization status and appropriate antibiotic therapy for nosocomial bacteremia caused by antibiotic-resistant gram-negative bacteria in an intensive care unit. Infect Control Hosp Epidemiol 2005; 26: 575-579.

27 Depuydt P, Benoit D, Vogelaers D, et al. Outcome in bacteremia associated with nosocomial pneumonia and the impact of pathogen prediction by tracheal surveillance cultures. Intensive Care Med 2006; 32: 1773-1781.

28 Mokart D, Guery BP, Bouabdallah R, et al. Deactivation of alveolar macrophages in septic neutropenic ARDS. Chest 2003; 124: 644-652.

29 Wheeler AP, Bernard GR. Acute lung injury and the acute respiratory distress syndrome: a clinical review. Lancet 2007; 369: 1553-1564.

30 Lecuyer L, Chevret S, Thiery G, et al. The ICU trial: a new admission policy for cancer patients requiring mechanical ventilation. Crit Care Med 2007; 35: 808-814. 